\title{
RELATO DE EXPERIÊNCIA: PROCESSO DE AVALIAÇÃO PSICOLÓGICA EM UM CENTRO DE REFERÊNCIA DE ASSISTÊNCIA SOCIAL (CRAS)
}

DOI: 10.22289/2446-922X.V7N1A19

\author{
Pedro Henrique Silva de Alencar \\ Noé do Carmo Bezerra \\ Karoline Giele Martins de Aguiar ${ }^{1}$
}

\section{RESUMO}

$\mathrm{A}(0)$ psicóloga(o) do CRAS trabalha com a prevenção e o fortalecimento de vínculos, seja de maneira individual ou coletiva, no contexto de vulnerabilidade social a partir de intervenções que promovam a proteção básica, pautada(o) no pensamento ético que garanta a efetivação de direitos das pessoas. O estudo trata-se de relato de experiência de estágio em Avaliação Psicológica e Psicodiagnóstico desenvolvido por acadêmicos do $7^{0}$ período do curso de Psicologia. Objetivou discutir demanda de Avaliação Psicológica, na atividade do profissional de Psicologia em Políticas Públicas assistenciais. O estágio ocorreu entre os meses de agosto a dezembro de 2020 em um Centro de Referência de Assistência Social de um bairro periférico, em uma cidade no interior do estado do Maranhão, após a autorização do Ministério da Educação (MEC), para o retorno dos estágios curriculares ocasionada pelas contingências do COVID-19. A experiência da realização do estágio em avaliação psicológica em uma instituição pública como o CRAS é desafiador para qualquer estudante de Psicologia, pelo fato da instituição não disponibilizar ambiente físico, estrutural e instrumental apropriado para o processo de avaliação psicológica, e obter-se êxito ao rastrear usuários do serviço que necessitavam de intervenções psicológicas e foram encaminhados aos serviços de saúde.

Palavras-Chave: Estágio; Políticas Públicas; Avaliação Psicológica; Psicologia e Assistência Social.

\section{EXPERIENCE REPORT: PSYCHOLOGICAL EVALUATION PROCESS IN A REFERENCE AND SOCIAL ASSISTANCE CENTER (CRAS)}

\section{ABSTRACT}

The CRAS psychologist works with the prevention and strengthening of bonds, whether individually or collectively, in the context of social vulnerability based on interventions that promote basic protection, based on ethical thinking that guarantees the realization of people's rights. The study is a report of an internship experience in Psychological Assessment and Psychodiagnosis developed

\footnotetext{
${ }^{1}$ Endereço eletrônico de contato: karol.giele@hotmail.com

Recebido em 13/03/2021. Aprovado pelo conselho editorial para publicação em 14/05/2021.
} 
by academics from the 7th period of the Psychology course, aiming to discuss the practice of the Psychological Assessment process, in the activity of the Public Policy psychology professional assistants. The internship took place between the months of August and December 2020 at a Reference Center for Social Assistance in a peripheral neighborhood in a city in the interior of the state of Maranhão, after authorization from the Ministry of Education - MEC, for the return of curricular internships. caused by COVID-19 contingencies. The experience of conducting the internship in psychological assessment in a public institution like CRAS is challenging for any student of psychology, since the institution does not provide the appropriate physical, structural and instrumental environment for the psychological assessment process, being successful in tracking users of the service that needed psychological interventions and were referred to health services.

Keywords: Internship; Public policy; Psychological Assessment; Psychology and Social Assistance.

\section{INFORME DE EXPERIENCIA: PROCESO DE EVALUACIÓN PSICOLÓGICA EN UN CENTRO DE REFERENCIA DE ASISTENCIA SOCIAL (CRAS)}

\section{RESUMEN}

EI psicóloga CRAS trabaja con la prevención y fortalecimiento de los lazos, ya sea de manera individual o colectiva, en el contexto de la vulnerabilidad social a partir de intervenciones que promueven la protección básica, basada en un pensamiento ético que garantiza la realización de los derechos de las personas. El estudio es un informe de una experiencia de pasantía en Evaluación Psicológica y Psicodiagnóstico desarrollada por académicos del $7 \mathrm{mo}$ período de la carrera de Psicología, con el objetivo de discutir la práctica del proceso de Evaluación Psicológica, en la actividad de los asistentes profesionales de psicología de Políticas Públicas. La pasantía se llevó a cabo entre los meses de agosto y diciembre de 2020 en un Centro de Referencia de Asistencia Social en un barrio periférico de una ciudad del interior del estado de Maranhão, previa autorización del Ministerio de Educación - MEC, para la devolución de currículum. pasantías causadas por contingencias COVID-19. La experiencia de realizar la pasantía en evaluación psicológica en una institución pública como CRAS es un desafío para cualquier estudiante de psicología, ya que la institución no brinda el entorno físico, estructural e instrumental adecuado para el proceso de evaluación psicológica, siendo exitoso en el seguimiento de los usuarios de el servicio que necesitó intervenciones psicológicas y fueron remitidos a los servicios de salud.

Palavras clave: Prácticas; Políticas públicas; Evaluación psicológica; Psicología y Asistencia Social.

\section{INTRODUÇÃO}

A Avaliação Psicológica (AP) pode ser compreendida como o processo de intervenção que envolve uma perspectiva sistematizada e estruturada de princípios teóricos, metodológicos e técnicos de investigação da personalidade e funções cognitivas. Este processo organiza-se a partir da coleta e levantamento de informações, envolvendo fontes fundamentais e complementares, validando a fidedignidade do método (CFP, 2018). 
De acordo com Löhr (2011) a AP é uma técnica exclusiva da(o) psicóloga(o) e, atualmente, trata-se de um dos principais campos de atuação deste profissional. A AP pode e deve ser uma ferramenta de trabalho da psicóloga(o) nos diversos contextos: clínicas particulares, consultórios, como ferramenta de diagnóstico e tratamento de pacientes; em escolas, na avaliação de componentes cognitivos e emocionais de crianças e adolescentes e jovens; em empresas, nos processos de seleção de pessoal; no trânsito, na avaliação das funções cognitivas e de personalidade de candidatos a condutores de veículos automotivos; avaliação no pré e pós procedimentos cirúrgicos sendo que, em alguns contextos, a AP é compulsória (CFP, 2019).

A Resolução n09/2018, determina que no processo de AP deve conter fontes fundamentais de informação para a sua legitimidade e, dentre as fontes fundamentais de informação mais utilizadas estão os testes psicológicos, sendo a sua aplicação, mensuração e análise de uso exclusivo da(o) psicóloga(o). Desde os primeiros testes psicológicos, no início do século passado, até a atualidade, com todos os critérios éticos e técnicos, a AP é ferramenta de trabalho do profissional de Psicologia (Noronha \& Reppold, 2010).

No entanto, a temática tem movimentado o cenário nacional atual, por decisão do Supremo Tribunal Federal (STF), que votou pela Ação Direta de Inconstitucionalidade (ADI) 3481 do inciso III, parágrafos 1 e 2 do Art. 18 da Resolução n 002/2003 do CFP, autorizando a utilização e comercialização de testes psicológicos por outras categorias profissionais. O Conselho Federal de Psicologia (CFP), ingressou no mês de abril de 2021, com medida judicial cautelar incidental, junto ao STF, e vem movimentando o diálogo e atualizando as informações pertinentes à categoria através das mídias sociais.

Diante do cenário atual, tornam-se imperativas discussões e debates sobre a AP, assim como a expansão e ampliação desta área de conhecimento pertinente à ciência psicológica. Diante das áreas de atuação já mencionadas, nas quais a AP já se confirma como instrumento necessário para prática do profissional de Psicologia, outros contextos como de atuação da(o) psicóloga(o), dentre eles, as Políticas Públicas suscitam melhor compreensão. Os autores Capitão, Scortegagna e Baptista (2005) apontam a importância da AP nas políticas públicas de saúde. Dessa forma, tornase prudente a discussão voltada para as políticas públicas de assistência social.

O Sistema Único de Assistência Social (SUAS), previsto na Lei Orgânica de Assistência Social (LOAS), é uma proposta de organização de políticas públicas federais voltadas para a assistência social e sua difusão sobre todo o território nacional. Suas ações são organizadas em dois segmentos: 1) Proteção Social Básica, com foco na prevenção de vulnerabilidade social através de projetos e benefícios específicos; e 2) Proteção Social Especial prestando serviços voltados para situações em que já houve quebra de direitos e indivíduos em situação de risco (Brasil, 2015).

Rev. Psicol Saúde e Debate. Mai., 2021:7(1): 268-279. 
O Centro de Referência de Assistência Social (CRAS), que está inserido como um dos programas do primeiro segmento, o de Proteção Social Básica, é um sistema público de promoção de serviços assistenciais preventivos de combate a situações de vulnerabilidades sociais, funcionando como principal dispositivo da proteção básica oferecida pelo SUAS (Brasil, 2009).

A partir destas novas organizações nacionais de políticas públicas voltadas para a assistência social, muitos profissionais puderam ser incorporados às equipes que integram os serviços e um deles é a(o) psicóloga(o). Portanto, a(o) psicóloga(o) no contexto assistencial atual tem assistido novas possibilidades de atuação e, ainda, acrescentado as técnicas oriundas de sua ciência nos campos de trabalho no qual está sendo inserido (Costa \& Cardoso, 2010).

Deste modo, o arcabouço teórico-científico da(o) psicóloga(o), dentro do CRAS, pode variar de acordo com o repertório específico de sua atuação prática, em conjunto com os saberes multidisciplinares, interdisciplinaridade e o diálogo como ferramentas de construção da compreensão da realidade (CFP, 2008). A AP, em usuários do CRAS, pode ser uma técnica da(o) psicóloga(o) para auxiliar na promoção de autonomia e saída dos indivíduos do contexto de vulnerabilidade social. O conceito de vulnerabilidade abordado diz respeito a pessoas que vivem em situação socioeconômica precária e/ou com fragilidades dos vínculos familiares ou comunitários precária ou de fragilidades (Pereira \& Guareschi, 2017). Pois permitirá uma compreensão global do ser humano, obtendo-se informações mais claras e precisas do usuário avaliado, facilitando os possíveis encaminhamentos e orientações advindas da AP.

Segundo Silva e Corgozinho (2011) o objetivo de todo serviço psicológico, junto às comunidades em situação de vulnerabilidade social assistidas pelo CRAS, é propiciar o desenvolvimento de autonomia desses sujeitos criando possibilidades que os auxiliem a sair das situações de risco em que se encontram.

Relacionado a essa ideia, a(o) psicóloga(o) do CRAS trabalha para minimizar aos indivíduos, seja de maneira individual ou coletiva, o contexto de vulnerabilidade social a partir de intervenções que promovam a proteção básica, pautada(o) no pensamento ético que garanta a efetivação dos direitos das pessoas (CFP, 2008).

Estudos realizados por Oliveira, Dantas, Solon, \& Amorim, (2011) e Oliveira et. al. (2014), o primeiro com 18 psicólogas(os), na região metropolitana de Natal no estado do Rio Grande do Norte e o segundo com 15 psicólogas(os) em municípios do interior do estado do Rio Grande do Norte, atuantes em CRAS apontam os serviços prestados com maior frequência por esses profissionais: acolhimento, entendido como a primeira atividade oferecida ao usuário que chega à instituição, que pode variar entre identificação das demandas dos usuários até possíveis encaminhamentos; atividades socioeducativas, como em grupos terapêuticos, oficinas, capacitação de profissionais e palestras em escolas; e, também, outros serviços surgem com frequência, entre os quais destacam-se visitas domiciliares e acompanhamento familiar. Macedo et al. (2018) 
corroboram os dados apresentados, pesquisa com 8 psicólogas(os), indicam as práticas exercidas de acolhimento, atendimento e orientação individual, encaminhados. A prática de AP não é mencionada como instrumento de trabalho nos estudos apresentados.

Considerando-se a importância da AP para a compreensão dos aspectos comportamentais e de personalidade, que definem uma pessoa, e que essa técnica é precípua na prática da(o) psicóloga(o) em qualquer contexto de atuação, faz-se importante discutir e esclarecer as possibilidades e desafios da utilização do processo de AP neste contexto, junto aos usuários do CRAS. Vislumbrando-se a discussão a partir da prática da AP como ferramenta de diagnóstico e intervenção nas Políticas Públicas, com indivíduos em vulnerabilidade social.

\section{MATERIAS E MÉTODOS}

Trata-se de um relato de experiência, desenvolvido por 6 acadêmicos do $7^{\circ}$ período do curso de Psicologia, no Estágio Básico de Avalição Psicológica e Processos de Diagnóstico, sob a orientação da supervisora da IES. O período de realização ocorreu entre agosto a dezembro de 2020 após a autorização do Ministério da Educação (MEC), para o retorno dos estágios curriculares ocasionado pelas contingências do COVID-19, deu-se no Centro de Referência de Assistência Social, de bairro periférico em uma cidade do interior do estado do Maranhão. A duração do estágio foi de 6 meses, os atendimentos deram-se de forma individual e semanal. Totalizando 6 usuários atendidos, através da busca espontânea para a submissão no processo de AP, a partir de informe disponibilizado no mural da instituição.

Os procedimentos realizados foram de 6 a 8 sessões com duração média de uma hora e perpassou as seguintes etapas: acolhimento, explanação e assinatura do Termo de Consentimento Livre e Esclarecido (TCLE), entrevista inicial e anamnese, testagem psicológica - utilizados testes de personalidade, testes de memória e rastreadores de ansiedade; de acordo com a demanda dos usuários e, por fim, entrevista devolutiva, momento de finalização do processo de AP e realização dos encaminhamentos necessários. Todos os atendimentos foram registrados pelos estagiários no prontuário do CRAS e também foi aberto o prontuário na instituição de ensino com os procedimentos realizados.

O relato segue as diretrizes e os preceitos da Resolução $n^{\circ}$ 510/2016, do Conselho Nacional de Saúde (CNS, 2016), com a aprovação de Comitê de Ética em Pesquisa, sob o Parecer no 4.614.249.

\section{RELATO DA EXPERIÊNCIA}

Rev. Psicol Saúde e Debate. Mai., 2021:7(1): 268-279. 
O estágio de AP transcorreu conforme o plano de trabalho organizado e planejamento dos estagiários e da disciplina, com o total de seis indivíduos atendidos. As demandas iniciais apresentadas eram relativas a três eixos: autoconhecimento, dificuldades relacionadas à família e à memória. Observou-se a assiduidade dos usuários aos atendimentos, colaboração e participação ativa no decorrer de todo o processo de AP e nas intervenções efetuadas. Fato essencial para que o estágio transcorresse de acordo com o plano, promovendo e provocando envolvimento dos estagiários, principalmente no processo de testagem psicológica e na entrevista devolutiva. Cerioni e Herzberg (2016) salientam a importância da adesão do paciente durante o processo, uma vez que a sua implicação durante a intervenção proporciona a redução de faltas e autonomia desde o início até a finalização do acompanhamento psicoterapêutico.

Quanto às competências dos estagiários que foram praticadas durante o processo, autores como Whitbourne e Halgin (2015) chamam a atenção para o respeito que a(o) psicóloga(o) deve ter para com o paciente, sobretudo, para evitar reduzi-lo a possíveis patologias ou, ainda, a ocorrer desistência durante o atendimento psicológico. Baseando seu trabalho no exercício constante da empatia como instrumento fundamental para qualquer estabelecimento de vínculo terapêutico, a(o) psicóloga(o) consegue realizar com efetividade as técnicas planejadas.

Seguindo o proposto por Whitbourne e Halgin (2015), a empatia é uma das habilidades fundamentais para o desempenho prático e ético do processo de estágio. Desde os primeiros até os últimos atendimentos os estagiários praticaram tal habilidade, através de escuta participativa e interessada na narrativa e nas demandas dos indivíduos, buscando descobrir os motivos subjetivos e latentes, a demanda inicial perpassava autoconhecimento, relações familiares ou sofrimento ocasionado pela pandemia. No conceito de latência abordado compreende-se esta como mecanismo psíquico atuante para organização e ampliação dos recursos mentais, evocados por demandas externas, que necessitem o ordenamento de demandas internas (Urribarri, 1999).

Prezou-se pela não interrupção do discurso, deixando-os livres para se expor à sua maneira e da forma que considerassem mais adequada. A prevalência das demandas levantadas referia-se à ansiedade, como parte pregressa de condição de saúde e dois dos usuários avaliados tinham histórico de intervenção medicamentosa, mas ambos não souberam informar se houve diagnóstico médico ou apenas a amenização dos sintomas por meio farmacológico. O estudo realizado por Barros et al. (2020), abrangendo todo território nacional com amostra de mais de 45.161 participantes evidenciou que $52,6 \%$ dos entrevistados relataram sintomas recorrentes de ansiedade ou nervosismo, nos últimos tempos, associados ao isolamento social ocasionado pelas restrições sociais para o combate ao COVID-19.

Os sintomas relacionados à ansiedade eram anteriores ao processo de restrição social, porém, a manifestação não ocasionava sofrimento psíquico ou prejuízos aos usuários avaliados. Com as medidas restritivas associadas ao isolamento social como forma de contenção da 
disseminação do Coronavírus (Sars Cov 2), propiciou a intensificação dos sintomas nos avaliados. Desta forma os estagiários basearam-se então em acolher a demanda, levantar informações referentes ao histórico de vida e história pregressa dos sintomas de ansiedade, buscando a compreensão da suspeita de quadros de transtornos de ansiedade ou ansiedade ocasionados pela atual imobilidade social, dentre outras variáveis que poderiam contribuir para a instalação do sofrimento psíquico. Todas as etapas foram fundamentais para a avaliação psicológica e configuram-se como imprescindíveis nos encaminhamentos e possíveis tratamentos dispensados ao paciente (Musskopf \& Lang, 2014).

A pandemia do Coronavírus (Sars Cov 2), além de ter causado grandes problemas sanitários, econômicos, políticos e sociais à humanidade, também provocou impactos negativos sobre a saúde mental das populações. Trabalhos recentes (Barros et al., 2020; Maia \& Dias, 2020) afirmam que a ansiedade é um dos transtornos mentais mais comuns surgidos a partir do contexto de isolamento social, caracterizada, principalmente, por medo de contrair a doença ou mesmo de morrer ou perder parentes e amigos; as circunstâncias afetaram de forma expressa a saúde mental de diversas pessoas. Sendo uma possibilidade, o suporte psicossocial disponibilizado pelo CRAS tende a fomentar estratégias de promoção de saúde mental e bem-estar para as pessoas e pode configurar-se como medidas urgentes e necessárias atualmente (Silva, Santos, \& Oliveira, 2020).

Durante o processo de AP, os indivíduos assistidos apresentaram facilidade em narrar suas queixas, demandas e angústias, não ocorrendo episódios de desorganização mental ou tristeza profunda. Contudo, o estágio, seguiu as normas de biossegurança, mesmo assim, observou-se comportamentos repetitivos de higienização das mãos com álcool em gel, recurso utilizado pelos indivíduos com intuito de manter o equilíbrio emocional, ao lidar com a situação pandêmica e a exposição a ambientes coletivos.

As experiências de vivência de sintomas de ansiedade, pelos usuários avaliados, foram ao encontro à experiência de ansiedade dos estagiários, pois, ao depararem-se aos sintomas do outro, puderam experimentar as próprias manifestações, mostrando que ambos os sujeitos, usuários e estagiários, fazem parte dessa mesma história. Em estudo de revisão sistemática sobre ansiedade em profissionais de saúde, confirma-se o risco elevado do surgimento de sintomas de ansiedade nesses profissionais, porém, no mesmo período de tempo, a prevalência de ansiedade identificada nos profissionais de saúde é semelhante à da população em geral (Silva, Cobucci, Soares, Lima, \& Andrade, 2021).

A percepção dos estágios sobre os sintomas iniciou pela observação do comportamento verbal - a taquilalia, fala excessiva e rápida, e comportamento de inquietação, não hesitando em responder com detalhes todas as perguntas. Esses sinais manifestos na maioria os indivíduos, segundo a literatura, podem ser indícios de traços de um transtorno de ansiedade (Dalgalarrondo, 2019).

Rev. Psicol Saúde e Debate. Mai., 2021:7(1): 268-279. 
Embora a finalidade do estágio em AP não tenha sido o de promover um processo psicoterapêutico, buscou-se durante os atendimentos oferecer aos indivíduos intervenções terapêuticas, sendo uma das competências e habilidades que norteiam a prática clínica da(o) psicóloga(o). Observou-se que, conforme transcorreram os atendimentos, os indivíduos apresentaram entusiasmo em continuar com os procedimentos sugeridos. Isso pode ser compreendido como forma de lidar com as situações e rotinas do dia a dia diante do enfrentamento da pandemia. Tais consequências foram possibilitadas através do desenvolvimento da aliança terapêutica entre os estagiários e os indivíduos atendidos, assim a aliança proposta desenvolve-se e se sustenta através do encontro entre profissional e pessoa atendida (Oliveira \& Benetti, 2015).

O estágio configurou-se como uma realidade singular para os estagiários, a qual propiciou o primeiro contato com a prática da ciência psicológica, durante a graduação. Refletindo, assim, desenvolver a práxis dos conhecimentos essenciais para a formação dos estagiários. Faz-se importante que essas habilidades sejam cada vez mais discutidas e treinadas pelos professores e supervisores, a fim de melhorar o desempenho do estagiário e evitar comportamentos ansiosos durante o estágio (Cerioni \& Herzberg, 2016).

Embora a(o) psicóloga(o) cada vez mais tem adentrado em espaços públicos, no contexto das políticas públicas de assistência social, existem muitos desafios para que este profissional atue de forma efetiva no CRAS. Conforme aponta Macêdo (2018), houve avanço na atuação da(o) psicóloga(o), porém a prática da clínica tradicional e a avaliação psicossocial precisam ser evidenciadas.

Os desafios da prática aparecem nas condições precárias de trabalho, em que falta de materiais e/ou recursos físicos e tecnológicos impossibilitam a completude da execução das atividades diárias e, ainda, o desconhecimento de outras categorias de profissionais que constituem a equipe de trabalho sobre o papel a ser executado pela(o) psicóloga(o) na instituição, restringindose ao atendimento psicológico. Essas dificuldades atrapalham a técnica, contudo, podem possibilitar a criação de novos dispositivos de prática nesses contextos (Macedo et al., 2018; Oliveira et al., 2014).

Autores como Flor e Goto (2015) alertam, ainda, para a falta de disciplinas teóricas e práticas específicas de psicologia social na grade curricular dos estudantes de Psicologia, o que pode dificultar sua inserção em postos de trabalho neste campo, no futuro, e na clareza do que se espera do trabalho psicológico visto por outros profissionais.

A finalização do estágio deu-se com o desfecho da entrevista devolutiva, e, neste momento, os estagiários vivenciaram sentimentos de angústia, medo, dúvida de não conseguir conduzir o momento e prepará-los para o encerramento; nessa sessão foram realizadas as instruções para os encaminhamentos necessários a outras instituições da Rede de Atenção Psicossocial - RAPS, como Centro de Atenção Psicossocial - CAPS. A prevalência dos resultados associados aos 
sintomas ansiosos é entendida pelos estagiários como reflexo do cenário atual do país. O processo de aprendizagem, neste cenário, provocou discussões referentes à diversidade de demandas acolhidas na instituição e a importância do processo de AP na prática da(o) psicóloga(o) como instrumento de investigação e condução dos casos.

A posição empática dos estagiários, durante a AP, gerou envolvimento com o estado de sofrimento atual e futuro dos usuários avaliados, oportunizando a sensibilização dos atendidos, favorecendo o protagonismo e a melhora do quadro sintomatológico atual. A escuta das expectativas é fator significativo para adesão ao processo psicoterapêutico. Não sendo único fator, mas também possibilita a maior adesão aos encaminhamentos indicados (Cerioni \& Herzberg, 2016).

\section{CONSIDERAÇÕES FINAIS}

A experiência da realização do estágio em AP em instituição pública como o CRAS foi desafiador e provocador de diversas reflexões, tais como políticas, sociais, formação acadêmica e da prática profissional da(o) psicóloga(o), permeada pelas dificuldades estruturais encontradas em diversas instituições públicas do país, a precariedade da edificação, as barreiras impostas pela instituição, a falta de recursos físicos e materiais para a execução das atividades, em contraste às exigências técnicas e éticas solicitadas ao processo de AP, como o sigilo profissional, o ambiente necessário para testagem e as condições psíquicas do próprio avaliador. Todos esses fatores mencionados podem comprometer validade e precisão dos instrumentos utilizados e a fidedignidade do processo de AP, afetando desde os resultados obtidos à condução psicoterapêutica que embasará o processo futuro.

Cabe, portanto, aos estagiários a utilização de estratégias norteadoras que organizem e desmontem os desafios encontrados a fim de favorecer os objetivos estabelecidos pelo estágio, ocasionando a aquisição de conhecimento necessário à formação. E o mesmo também é esperado no tratamento ofertado ao usuário, ressaltando a postura ética profissional solicitada a ser reinventada diante dos desafios. Deste modo, ainda que as condições das instituições CRAS possam obstruir a realização da AP aos seus usuários, cabe ao profissional de Psicologia executar e conduzir a AP com as premissas ética de compromisso e de responsabilidade social. Acredita-se que o desenvolvimento de pesquisas empíricas possa fomentar a ampliação das discussões atuais, promovendo a inserção do processo de AP nas políticas públicas do SUAS, como instrumento de intervenção e investigação das demandas absorvidas pelo profissional de Psicologia.

Rev. Psicol Saúde e Debate. Mai., 2021:7(1): 268-279. 


\section{REFERÊNCIAS}

Barros, M. B. D. A., Lima, M. G., Malta, D. C., Szwarcwald, C. L., Azevedo, R. C. S. D., Romero, D., ... \& Gracie, R. (2020). Relato de tristeza/depressão, nervosismo/ansiedade e problemas de sono na população adulta brasileira durante a pandemia de COVID-19. Epidemiologia e Serviços de Saúde, 29, e2020427. Recuperado em 08 de março de 2021 de: https://doi.org/10.1590/S1679-49742020000400018

Brasil, (1993). Dispõe sobre a organização da Assistência Social e dá outras providências. Lei $n^{\circ}$ 8.742. Lei Orgânica de Assistência Social (LOAS). Brasília: DF, 7 de dezembro de 1993. Recuperado em 08 de março de 2021 de: https://www.mds.gov.br/webarquivos/publicacao/assistencia_social/Normativas/LoasAnotada. pdf

Brasil. (2009). Ministério do Desenvolvimento Social e Combate à Fome. Orientações Técnicas: Centro de Referência de Assistência Social - CRAS. Brasília: 2009. Recuperado em 09 de março de 2021 de: http://www.mds.gov.br/webarquivos/publicacao/assistencia_social/Cadernos/orientacoes_Cra s.pdf.

Brasil. (2015) Ministério da Cidadania. Sistema Único de Assistência Social - Suas. Brasília - DF, (2015). Recuperado em 08 de março de 2021 de: https://www.mds.gov.br/webarquivos/publicacao/assistencia_social/Livros/RedeSuas_gestaoe sistema.pdf

Brasil. Ministério da Saúde. Conselho Nacional de Saúde. Resolução no 510, de 7 de abril de 2016. Trata sobre as diretrizes e normas regulamentadoras de pesquisa em ciências humanas e sociais. Diário Oficial da União, Brasília, DF, 24 maio 2016. Recuperado em 18 de abril de 2021. https://bvsms.saude.gov.br/bvs/saudelegis/cns/2016/res0510_07_04_2016.html

Capitão, C. G., Scortegagna, S. A., \& Baptista, M. N. (2005). A importância da avaliação psicológica na saúde. Avaliaçao Psicologica: Interamerican Journal of Psychological Assessment, 4(1), 7582. Recuperado em 18 de abril de 2021: file:///C:/Users/SAMSUNG/Downloads/DialnetAlmportanciaDaAvaliacaoPsicologicaNaSaude-6674821\%20(1).pdf

Cerioni, R. A. N.; Herzberg, E. (2016) Expectativas de Pacientes acerca do Atendimento Psicológico em um Serviço-Escola: da Escuta à Adesão. Psicologia: Ciência e Profissão, v. 36 n 3, p. 597609, jul.-set., 2016. Recuperado em 08 de março de 2021 de: DOI: 10.1590/19823703001402014

Conselho Federal de Psicologia. (2019). Avaliação Psicológica Compulsória. Brasília, CFP, 2019. Recuperado em 18 de abril de 2021: https://satepsi.cfp.org.br/docs/1910-DialogosEd10Encarte.pdf

Conselho Federal de Psicologia, (2018). Resolução $n^{\circ} 9$ de 25 de abril de 2018. Brasília, 2018. Recuperado em 08 de março de 2021 de: https://crpsc.org.br/noticias/cfp-publica-novaresolucao-sobre-avaliacao-psicologica

Conselho Federal de Psicologia, (2003). Resolução $n^{\circ} 02$ de 24 de março de 2003. Brasília, 2003. Recuperado em 08 de março de 2021 de: https://site.cfp.org.br/wpcontent/uploads/2003/03/resolucao2003_02_Anexo.pdf

Rev. Psicol Saúde e Debate. Mai., 2021:7(1): 268-279. 
Conselho Federal de Psicologia. (2008). Referência técnica para atuação do(a) psicólogo(a) no CRAS/SUAS. Brasília, CFP, 2008. Recuperado em 08 de março de 2021 de: https://site.cfp.org.br/publicacao/referencias-tecnicas-para-atuacao-doa-psicologoa-no-crassuas/

Costa, A. F. de S.; Cardoso, C. L. (2010). Inserção do Psicólogo em Centros de Referência de Assistência Social - CRAS. Gerais: Revista Interinstitucional de Psicologia, v. 3, n. 2, p. 223229, Juiz de Fora: 2010. Recuperado em 09 de março de 2021 de: http://pepsic.bvsalud.org/pdf/gerais/v3n2/v3n2a11.pdf

Dalgalarrondo, P. (2019). Psicopatologia e Semiologia dos Transtornos Mentais. 3. ed. Porto Alegre: Artmed.

Flor, T. C.; Goto, T. A., (2015). Atuação do Psicólogo no CRAS: uma Análise Fenomenológicoempírica. Revista da Abordagem Gestáltica, v. 21, n. 1, janeiro-junho, p. 22-34, Goiânia: 2015. Recuperado em 09 de março de 2021 de: https://www.redalyc.org/pdf/3577/357742812004.pdf

Löhr, S. S. (2011). Avaliação psicológica na formação do profissional da Psicologia, algumas reflexões. In Conselho Federal de Psicologia, Ano da avaliação psicológica - Textos geradores (pp. 143-149). Brasília, DF: Conselho Federal de Psicologia

Maia, B. R., \& Dias, P. C. (2020). Ansiedade, depressão e estresse em estudantes universitários: o impacto da COVID-19. Estudos de Psicologia (Campinas), 37. Recuperado em 09 de março de 2021 de: http://dx.doi.org/10.1590/1982-0275202037e200067

Macedo, O. J. V., Lima, C. M. P. D., Brito, F. H. S. D., Souza, J. N. P. D., Sousa, N. K. M. D., Sousa, S. P. D., \& Dias, S. G. (2018). Atuação dos Profissionais de Psicologia nos CRAS do Interior da Paraíba. Trends in Psychology, 26(2), 1083-1097. Recuperado em 09 de março de 2021 de: http://dx.doi.org/10.9788/tp2018.2-20pt

Musskopf, G. M.; Lang, C. S. (2014). A importância do acolhimento aos pacientes que buscam atendimento psicológico no Instituto Integrado de Saúde. p. 473-486, maio. Recuperado em 09 de março de 2021 de: https://scholar.google.com.br/scholar?hl=ptR\&as_sdt $=0 \% 2 \mathrm{C} 5 \& \mathrm{q}=.+\mathrm{A}+\mathrm{import} \% \mathrm{C} 3 \% \mathrm{~A} 2 \mathrm{ncia}+\mathrm{do}+$ acolhimento+aos+pacientes+que+busca $\mathrm{m}+$ atendimento+psicol\%C3\%B3gico+no+Instituto+Integrado+de+Sa\%C3\%BAde.+\&btnG

Noronha, A. P. P.; Reppold, C. T. (2010) Considerações sobre a avaliação psicológica no Brasil. Psicologia, Ciência e Profissão, v. 30, p. 192-201, São Paulo: 2010. Recuperado em 09 de março de 2021 de: https://www.scielo.br/pdf/pcp/v30nspe/v30speca09.pdf.

Oliveira, N. H.; Benetti, S. P. da C. (2015). Aliança terapêutica: estabelecimento, manutenção e rupturas da relação. Arquivos Brasileiros de Psicologia, v. 67, n. 1. p. 125-138, Rio de Janeiro. Recuperado em 09 de março de 2021 de:https://www.redalyc.org/pdf/2290/229044976010.pdf.

Oliveira, I. F. D., Oliveira, N. L. D. A., Nascimento, M. N. C. D., Araújo, R. L., Coelho-Lima, F., \& Amorim, K. M. D. O. (2014). Atuação dos psicólogos nos CRAS do interior do RN. Psicologia \& Sociedade, 26(SPE2), 103-112. Recuperado em 09 de março de 2021 de: https://doi.org/10.1590/S0102-71822014000600011

Oliveira, I. F., Dantas, C. M. B., Solon, A. F. A. C., \& Amorim, K, M. O. (2011). A prática psicológica na proteção social básica do SUAS [Edição especial]. Psicologia \& Sociedade, 23, 140-149. doi: 10.1590/S0102-71822011000400017 
Pereira, V. T., \& Guareschi, P. A. (2017). A psicologia no CRAS: un estudo de representações sociais. Psicologia \& Sociedade, 29, 119584. Recuperado em 19 de abril de 2021 de: https://www.redalyc.org/pdf/3093/309350113001.pdf

Silva, D. F. O., Cobucci, R. N., Soares-Rachetti, V. D. P., Lima, S. C. V. C., \& Andrade, F. B. D. (2021). Prevalência de ansiedade em profissionais da saúde em tempos de COVID-19: revisão sistemática com metanálise. Ciência \& Saúde Coletiva, 26, 693-710.

Silva, H. G. N.; Santos, L. E. S. dos; Oliveira, A. K. S. de (2020). Efeitos da pandemia do novo Coronavírus na saúde mental de indivíduos e coletividades. Journal of Nursing and Health. Recuperado em 09 de março de 2021 de: https://periodicos.ufpel.edu.br/ojs2/index.php/enfermagem/article/viewFile/18677/11414.

Silva, J. V.; Corgozinho, J. P. (2011). Atuação do psicólogo, SUAS/CRAS e psicologia social comunitária: possíveis articulações. Psicologia \& Sociedade, v. 23, p.12-21, Belo Horizonte. Recuperado em 09 de março de 2021 de: https://www.scielo.br/pdf/psoc/v23nspe/a03v23nspe.pdf.

Urribarri, R. (1999). Descorriendo el velo: sobre el trabajo de la latencia. Revista de la Sociedad Colombiana de Psicoanálisis, 210-42.

Whitbourne, S. K.; Halgin, R. P. (2015). Psicopatologia: perspectivas clínicas dos transtornos psicológicos. 7. ed. Porto Alegre: AMGH.

Zimerman, D. E. (2009). Fundamentos psicanalíticos: teoria, técnica e clínica. Porto Alegre: Artmed. 